\title{
Prolonged QTc Interval Is an Electrophysiological Hallmark of Cirrhotic Cardiomyopathy
}

\author{
Adnan Bashir Bhatti*, Farhan Ali, Siddique Akbar Satti \\ Capital Development Authority (CDA) Hospital, Islamabad, Pakistan \\ Email: ${ }^{*}$ dr.adnanbashir@gmail.com
}

Received 26 November 2013; revised 26 December 2013; accepted 2 January 2014

Copyright (C) 2014 by authors and Scientific Research Publishing Inc.

This work is licensed under the Creative Commons Attribution International License (CC BY). http://creativecommons.org/licenses/by/4.0/

(c) (†) Open Access

\section{Abstract}

BACKGROUND: Cirrhotic Cardiomyopathy is a relatively ill-characterized condition, which is often under-diagnosed due to absence of defined diagnostic criteria. ECG showing corrected QT Interval prolongation is the most suitable available option for diagnosis of this condition. OBJECTIVE: To determine the frequency of corrected QT interval prolongation in patients with liver cirrhosis. METHODOLOGY: Patients $(n=166)$ with confirmed cirrhosis, 30 years or older, presented in the outpatient and emergency department of medicine at Capital Hospital Islamabad between 1 October 2011 and 30 September 2012, were enrolled in this cross-sectional study after taking consent. ECG was done using calibrated ECG machine, and the QT Interval was measured. Corrected QT was calculated using Bazett's formula and a QTc of more than 0.44 seconds was considered as being prolonged. RESULTS: The mean age of the patients was $57.05 \pm 12.03$ years. The corrected QT Interval varied from $337 \mathrm{~ms}$ to $560 \mathrm{~ms}$. The mean QTc Interval was $429.92 \mathrm{~ms} \pm 45.11$. QTc was prolonged in 41 out of 166 patients (24.7\%). Frequency of QTc prolongation was $4.5 \%$ in Child Pugh Grade A, 23.2\% in Child Pugh Grade B, and 32.0\% in Child Pugh Grade C. Association of Child Pugh Scoring with QTc prolongation was determined and found to be statistically significant $(P<0.05)$. CONCLUSION: QTC interval was prolonged in $24.7 \%$ of cirrhotic patients in our study. There was a significant increase in frequency with worsening of Child Pugh Grade, thereby indicating an association between QTc prolongation and the severity of cirrhosis.

\section{Keywords}

Cirrhosis; QT Interval; Cirrhotic Cardiomyopathy

\footnotetext{
${ }^{*}$ Corresponding author.
} 


\section{Introduction}

Liver cirrhosis refers to a progressive condition that disrupts the normal architecture of the liver, and can be caused by a number of conditions. Up to $90 \%$ of liver parenchymas undergo destruction before liver failure becomes clinically visible [1]. In developing countries, Hepatitis B and C have been described as the leading causes of cirrhosis, whereas in developed countries, Alcoholic liver disease (ALD) and Non-Alcoholic Steatohepatitis (NASH), in addition to Hepatitis C, have been named as the leading causes [2]. In the United States, liver cirrhosis is the $10^{\text {th }}$ leading cause of death for men, and the $12^{\text {th }}$ leading cause for women, and is responsible for approximately 27,000 deaths each year [3]. The majority of liver cirrhosis patients are male, with a male to female ratio ranging from 1.3:1 to 4:1 [2], the highest mortality rates observed in patients 60 - 70 years old $[4]$.

Cirrhotic Cardiomyopathy is the term used to describe a constellation of features indicative of abnormal heart structure and function, which are often secondary to liver cirrhosis [5] [6]. The effects of liver cirrhosis on the cardiovascular system include diastolic dysfunction, impaired stress response, structural changes, and electrophysiological abnormalities such as prolongation of repolarization time (QT interval prolongation). Various biochemical and electrophysiological markers have been identified to aid in the diagnosis of cirrhotic cardiomyopathy, such as raised Atrial Natriuretic Peptide (ANP) and B-Type Natriuretic Peptide (BNP) levels, elevated Cardiac Troponin I, and specific changes on Echocardiography. However, due to its widespread availability and cost-effectiveness, for many, the most suitable screening option is electrocardiography (ECG), which shows a prolonged corrected QT interval (QTc) in the case of cirrhotic cardiomyopathy [7]. QTc prolongation has been associated with an enhanced risk of a number of life-threatening cardiac arrhythmias, such as torsades de pointes (TdP) and ventricular fibrillation (VF), as well as with sudden cardiac death [8]. Frequency of QTc prolongation increases with a worse Child Pugh score, and it has been shown to positively correlate with a reduced heart rate variability, both of which are independent prognostic factors [9].

The rationale of this study was to identify the burden of cirrhotic patients with corrected QT interval prolongation in a Pakistani cohort, and to optimize their management and raise awareness levels among local and international health providers.

\section{Material and Methods}

\subsection{Data Collection Phase}

This study was conducted in the emergency and outpatient medical department of Capital Hospital Islamabad, Pakistan, which is tertiary care hospital, from 1 October 2011 to 30 September 2012. After taking permission from the Head of the Department of Medicine, Hospital Ethical Committee, the data collection phase was initiated.

By non-probability convenient sampling 166 patients with confirmed liver cirrhosis aged 30 years or older, were enrolled in the study by using the WHO Sample Size Calculator, with a confidence level of 95\%; population proportion 19.2\%; and an absolute precision of $6 \%$.

Verbal consent was taken from all patients after explaining the nature and purpose of the study at the beginning of the study. To minimize bias, all patients were handled by the same physician. Patients with confirmed cirrhosis were identified, and detailed medical histories were taken for diagnoses, and for the fulfillment of the required selection criteria.

\subsection{Methods}

Information was collected using structured proforma. Information regarding baseline characteristics of the patients was collected first, and their age and sex was noted. By vigorous and strict criteria only patients of confirmed cirrhosis were selected to be part of the study. Next, detailed medical histories were taken regarding symptoms of chronic liver disease. General physical examinations were performed for jaundice, skin rashes and vitals such as blood pressure, temperature, pulse rate and respiratory rate. Detailed examinations were done for liver size, ascities, and splenomegaly. Ultrasound scans were performed for all patients. Electrocardiography was done by using a calibrated ECG machine for all patients, to determine the presence or absence of QT interval prolongation. 


\subsection{Data Analysis}

The statistical package for social sciences (SPSS, version 16.0, Chicago, IL) was used to enter and analyze the data. Mean values, frequencies, percentages and P-values were calculated.

\subsection{Exclusion Criteria}

1) Patients who were suffering from any cardiac problem including ischemic heart disease, hypertension, conduction defects, and atrial fibrillation.

2) Patients taking drugs that may have prolonged the QT Interval, including calcium channel blockers, antihistamines, antipsychotics, macrolides, quinolones, and amiodarone.

3) Patients suffering from any other condition causing prolongation of QT interval.

\section{Results}

The age of the patients ranged between 33 years to 90 years. The mean age $( \pm \mathrm{SD})$ of the patients was 57.05 $( \pm 12.03)$ years, as depicted in Figure 1. Males were predominate, as seen in Figure 2. The distribution of patients according to Child Pugh is shown in Figure 3.

The corrected QT Interval varied from $337 \mathrm{~ms}$ to $560 \mathrm{~ms}$. The mean QTc Interval was $429.92 \pm 45.11 \mathrm{~ms}$. QTc was prolonged in 41 out of 166 patients, yielding a frequency of QTc prolongation due to cirrhosis to be 24.7\%, as shown in Figure 4. The frequency of QTc prolongation was more marked in the age group 46 - 60 years, as shown in Table 1. The Frequency of QTc prolongation was $26 \%$ in males, whereas for females, the frequency of QTc prolongation was 22.9\% (P > 0.05). The association of Child Pugh Scoring with QTc prolongation was more marked in Child Pugh Grade C (32.0\%) compared to Grade A and B, as shown in Table 2.

\section{Discussion}

The prevalence of cirrhosis has been demonstrated to be increasing in Pakistan, largely owing to the rapid spread of hepatitis B and C [10]. Cirrhosis is a leading cause of morbidity and mortality not only in Pakistan, but worldwide, and each year, thousands of patients are affected by this chronic condition [3]. Various factors have been shown to play a role in the spread of hepatitis B and C, including intravenous drug use with shared needles; unsterilized medical equipment; unscreened blood transfusions; having unprotected sex, and sharing personal items such as razors [11].

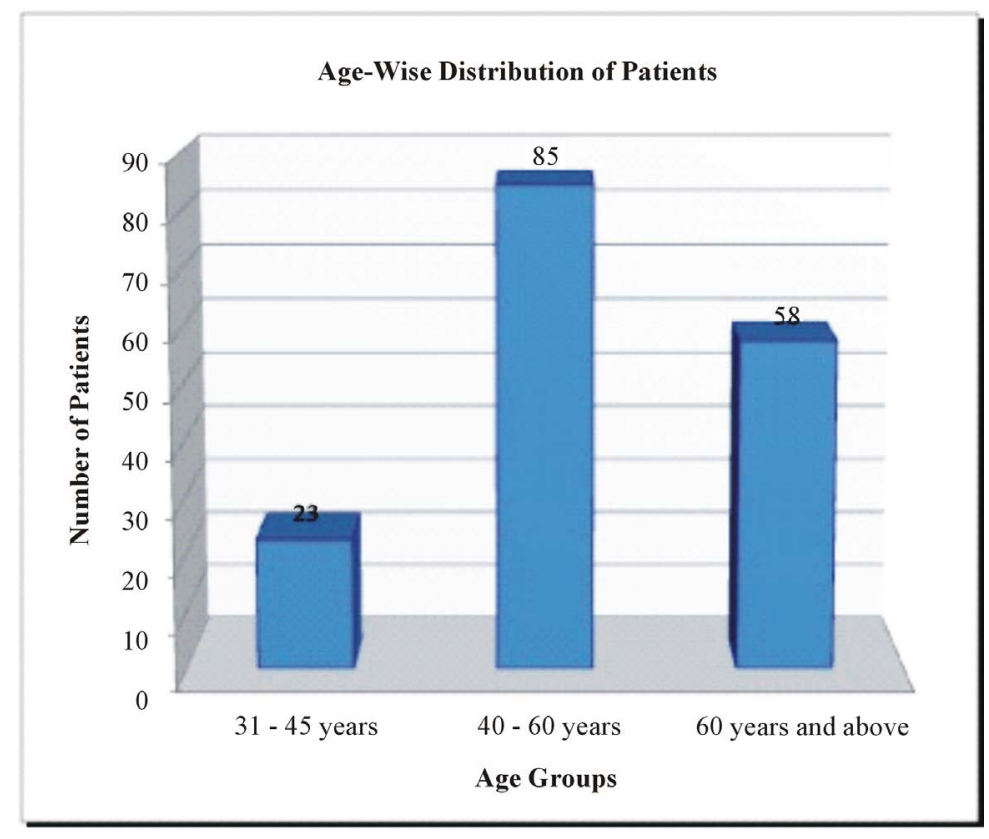

Figure 1. Age distribution of patients $(n=166)$. 


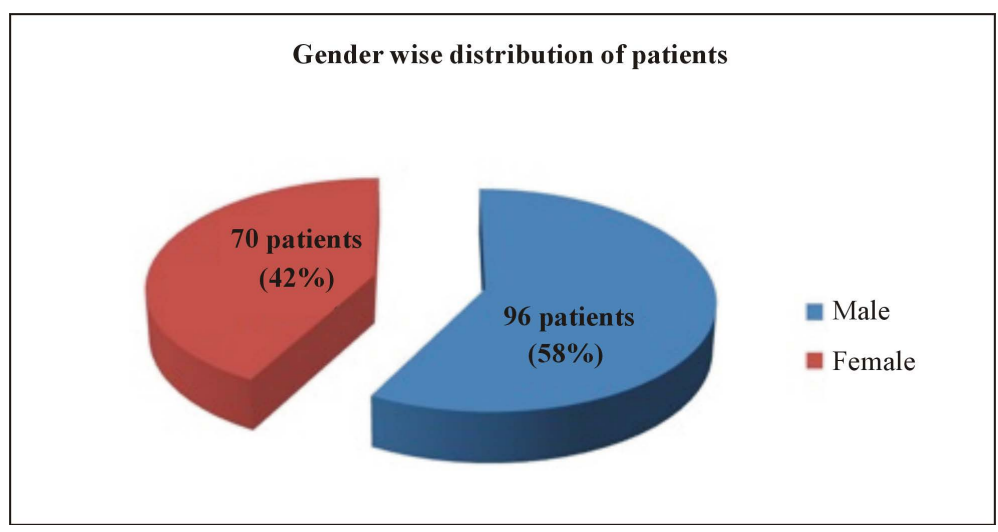

Figure 2. Sex distribution of patients $(n=166)$.

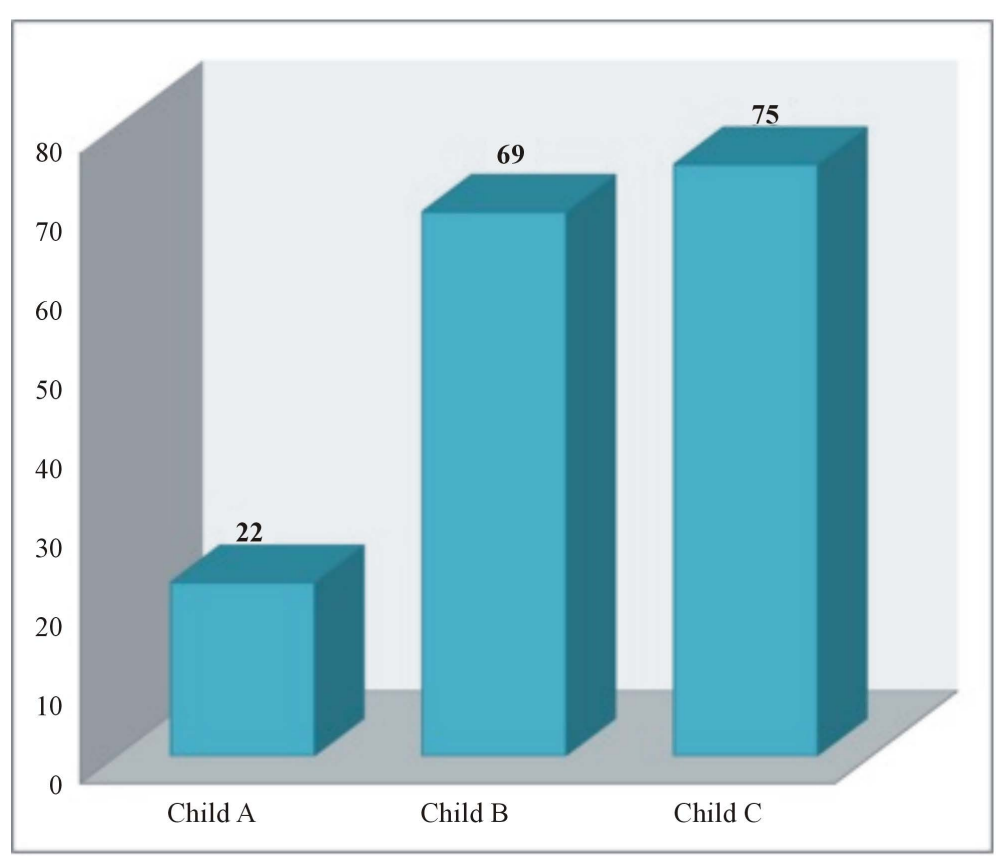

Figure 3. Distribution of cirrhosis patients according to Child Pugh Class (P > $0.05)$.

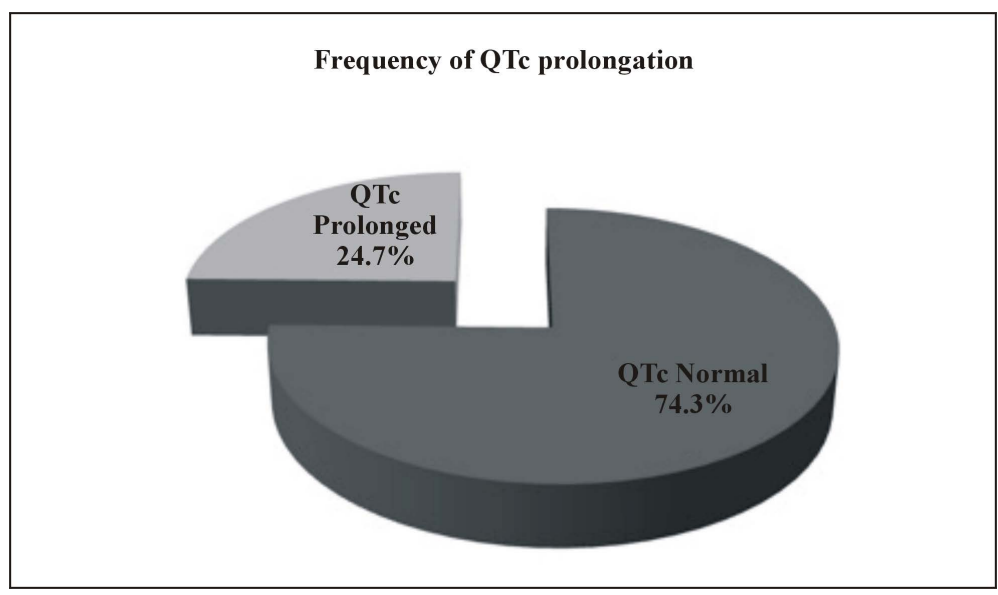

Figure 4. Frequency of QTc prolongation in 166 cirrhosis patients. 
Table 1. QT with regards sex $(\mathrm{P}>0.05)$.

\begin{tabular}{ccccc}
\hline \multirow{2}{*}{ QT } & \multicolumn{3}{c}{ Age Group } & \multirow{2}{*}{ Total } \\
\cline { 2 - 4 } & $\mathbf{3 1 - 4 5}$ & $\mathbf{4 6 - 6 0}$ & $>\mathbf{6 1}$ & \\
\hline \multirow{2}{*}{ Normal } & $\mathbf{( 1 3 . 9 \% )}$ & $\mathbf{( 5 1 . 2 \% )}$ & $\mathbf{( 3 4 . 9 \% )}$ & \\
& $(78.3 \%)$ & $(72.9 \%)$ & $(77.6 \%)$ & 125 \\
\multirow{2}{*}{ Prolonged } & 5 & 23 & 13 & \\
& $(21.7 \%)$ & $(27.1 \%)$ & $(22.4 \%)$ & 41 \\
Total & 23 & 85 & 58 & \multirow{2}{*}{166} \\
& $(100 \%)$ & $(100 \%)$ & $(100 \%)$ & \\
\hline
\end{tabular}

Table 2. QT with regards to Child Score $(\mathrm{P}<0.05)$.

\begin{tabular}{cccccc}
\multirow{2}{*}{ QT } & \multicolumn{3}{c}{ Child Score } & Total & P-value \\
\cline { 2 - 4 } & A & B & C & & \\
Normal & 21 & 53 & 51 & 125 & $<0.05$ \\
& $(95.5 \%)$ & $(76.8 \%)$ & $(68 \%)$ & & \\
Prolonged & 1 & 16 & 24 & 41 & \\
Total & $(4.5 \%)$ & $(23.2 \%)$ & $(32.0 \%)$ & & \\
\hline
\end{tabular}

A substantial proportion of patients with cirrhosis affected by ascites, volume overload, and signs of hyperdynamic circulation, have normal resting echocardiographic parameters but abnormal cardiac responses [12]. Cirrhotic cardiomyopathy is one relatively common cardiovascular complication arising secondary to liver cirrhosis, and despite an increased baseline cardiac output, cirrhotic patients have a suboptimal ventricular response to stress [12].

The pathogenesis of cirrhotic cardiomyopathy includes diminished beta-adrenergic receptor signal transduction; cardiomyocyte cellular plasma membrane dysfunction; and increased activity or levels of cardio-depressant substances such as cytokines, endogenous cannabinoids, and nitric oxide. Although cirrhotic cardiomyopathy is usually clinically silent, or not very severe, overt heart failure can be triggered by factors such as liver transplantation or transjugular intrahepatic portosystemic shunt insertion in these patients [13]. Currently, strict diagnostic criteria for cirrhotic cardiomyopathy are lacking, and hence, the disease often goes unrecognized [14].

Similarly to our study, Genovesi et al. showed in their study that most of the patients were $>45$ years of age (86.1\%), with the majority being in the $45-60$ year age group (51.2\%), indicating an increased prevalence of cirrhosis in higher age groups, which is understandable considering the long natural history of cirrhosis; it may take up to 30 years from the onset of infection to development of cirrhosis [15]. However, despite there being no significant difference between the patient age and sex in the two studies, Zuberi et al. reported a much lower mean age of 34.4 years in their study population [7] [15].

Our patients were distributed unevenly among Child Pugh Grade A, B and C, with the majority being in Grade B and C. This was not unexpected, since the samples were taken mostly from the inpatient department, and Child Pugh Grade A patients are admitted less frequently than grade B or C patients. Zuberi et al. also described a similarly skewed distribution of patients with more than half of them being in Child Pugh Grade C [7].

Li et al. demonstrated a mean QTc of $421 \pm 38 \mathrm{~ms}$ in their study [16], and Bernardi et al. also conducted a similar investigation and reported a mean QTc of $440 \pm 3.2 \mathrm{~ms}$ [9]. A study by Zuberi et al. demonstrated a mean QTc of $438 \mathrm{~ms} \pm 15 \mathrm{~ms}$ [7]. Our results were comparable with all of these studies.

The frequency of QTc prolongation in our study population was found to be $24.7 \%$. This is close to the results presented by Zuberi et al. in their study, in which they showed the frequency of QTc prolongation to be $19.2 \%$ [7]. However, other international studies have shown a wide range of values. It was found to be $46.2 \%$ in a study by Bernardi et al.; $46.93 \%$ in a study by Li et al.; Bal et al. demonstrated a QTc prolongation frequency of 56\% in their study; and Kosar et al. determined the frequency of QTc prolongation to be $32 \%$ in their study population [9] [16]-[18]. This discrepancy may be explained by the presence of other compounding factors such as electrolyte disturbances, concomitant cardiac problems, or use of QTc prolonging drugs, which were excluded in our study but might have been included in other studies. Moreover, the variable spread of severity of cirrhosis as 
shown by Child Pugh Score or any other model in all these studies would also have played a role in this wide range of results. We did not detect a difference in prevalence between males and females, as confirmed by these other studies as well [9] [16]-[18].

When distributed along the lines of Child Pugh Score, we observed a progressive increase in the frequency of QTc prolongation with the worsening of the Child Pugh Score, hence indicating a direct relationship between the stage of cirrhosis and the development of cirrhotic cardiomyopathy. In other words, the more advanced the cirrhosis, the greater the chances of developing cirrhotic cardiomyopathy and the associated symptoms. In their study, Li et al. reported a prolongation frequency of $21.05 \%, 56.89 \%$ and $59.60 \%$ among Child A, B and C, respectively, showing a rising trend [16]. Bernardi et al. demonstrated a similar increasing frequency with results of $25 \%, 51 \%$ and $60 \%$ respectively [9]. Our study showed similar results.

QTc is affected by a number of factors other than cirrhosis itself, such as electrolyte disturbances and certain drugs. These factors are usually present in patients of cirrhosis but were not accounted for in our study, so the frequency of QTc prolongation may be even higher when cirrhotics with these compounding factors are included in the study. The effect of etiology on the disease was not studied.

\section{Conclusion}

There was a statistically significant increase in frequency of QTc prolongation with worsening of Child Pugh Grade, thereby indicating an association between QTc prolongation and the severity of cirrhosis. There was no significant difference in frequency among males and females, or among different age groups, as seen in Figures 1 and 2. Patients with cirrhosis with QTc prolongation have a worse prognosis, as compared to patients with normal QTc duration.

\section{Recommendations}

1) Greater awareness among health professionals must be ensured regarding the presence of QTc prolongation in cirrhosis to avoid overlooking the condition in such patients.

2) ECG should be made an integral part of the work-up and follow-up of every patient with cirrhosis.

3) QTc interval measurement and rate correction should be taught to all health professionals, and its uses and implications should be explained thoroughly.

4) Electrolyte disturbances such as hypokalemia and hypomagnesimia, which can lead to further prolongation of QT interval, should be closely monitored and corrected as soon as possible, and such patients must avoid drugs that may cause these disturbances whenever possible.

5) All QT interval-prolonging drugs should be used very carefully and after considering the risk to benefit ratio in patients of cirrhosis, especially those with QTc prolonged.

6) Quinolones are a widely used drug group in cirrhosis, and they are known to cause QT prolongation. Further work on this subject is needed to assess the risk to benefit ratio of this group in cirrhosis, and alternative, safer medicines must be developed.

7) Beta-blockers have been shown to reduce the QT interval, and further studies are required to confirm this so that beta-blockers can be incorporated into the management of patients with prolonged QTc.

8) Special care must be taken in patients with QTc prolongation who develop SBP, or when undergoing TIPPS or Liver transplant.

\section{References}

[1] Heidelbaugh, J.J. and Bruderly, M. (2006) Cirrhosis and Chronic Liver Failure: Part I. Diagnosis and Evaluation. American Family Physician, 74, 756-62.

[2] Stroffolini, T., Sagnelli, E., Almasio, P., et al. (2004) Characteristics of Liver Cirrhosis in Italy: Results from a Multicenter National Study. Digestive and Liver Disease, 36, 56-60. http://dx.doi.org/10.1016/j.dld.2003.07.007

[3] Anderson, R.N. And Smith, B.L. (2003) Deaths: Leading Causes for 2001. National Vital Statistics Reports, 52, 1-85.

[4] Haliday, M.L., Coates, R.A. and Rankin, J.G. (1991) Changing Trends of Cirrhosis Mortality in Ontario, Canada 19111986. International Journal of Epidemiology, 20, 199-208. http://dx.doi.org/10.1093/ije/20.1.199

[5] Kowalski, H.J. and Abelmann, W.H. (1953) The Cardiac Output at Rest in Laennec's Cirrhosis. Journal of Clinical Investigation, 32, 1025-1033. http://dx.doi.org/10.1172/JCI102813 
[6] Abelmann, W.H., Kowalski, H.J. and McNeely, W.F. (1955) The Hemodynamic Response to Exercise in Patients with Laennec's Cirrhosis. Journal of Clinical Investigation, 34, 690-695. http://dx.doi.org/10.1172/JCI103120

[7] Zuberi, B.F., Ahmed, S., Faisal, N., et al. (2007) Comparison of Heart Rate and QTc Duration in Patients of Cirrhosis of Liver with Non-Cirrhotic Controls. Journal of the College of Physicians and Surgeons Pakistan, 17, 69-71.

[8] Chugh, S.S., Reinier, K., Singh, T., et al. (2009) Determinants of prolonged QT Interval and Their Contribution to Sudden Death Risk in Coronary Artery Disease: The Oregon Sudden Unexpected Death Study. Circulation, 119, 663670. http://dx.doi.org/10.1161/CIRCULATIONAHA.108.797035

[9] Bernardi, M., Calandra, S., Colantoni, A., et al. (1998) Q-T Interval Prolongation in Cirrhosis: Prevalence, Relationship with Severity, and Etiology of the Disease and Possible Pathogenetic Factors. Hepatology, 27, 28-34. http://dx.doi.org/10.1002/hep.510270106

[10] Walsh, K. and Alexander, G.J. (2001) Update on Chronic Viral Hepatitis. Postgraduate Medical Journal, 77, $498-505$. http://dx.doi.org/10.1136/pmj.77.910.498

[11] Smedsrod, B., Pertoft, H., Gustafson, S., et al. (1990) Scavenger Functions of the Liver Endothelial Cell. Biochemical Journal, 266, 313-327.

[12] Ripoll, C., Catalina, M.V., Yotti, R., et al. (2008) Cardiac Dysfunction during Liver Transplantation: Incidence and Preoperative Predictors. Transplantation, 85, 1766-1772. http://dx.doi.org/10.1097/TP.0b013e318172c936

[13] Alqahtani, S.A., Fouad, T.R. and Lee, S.S. (2008) Cirrhotic Cardiomyopathy. Seminars in Liver Disease, $28,59-69$. http://dx.doi.org/10.1055/s-2008-1040321

[14] Jessup, M., Abraham, W.T., Casey, D.E., et al. (2009) 2009 Focused Update: ACCF/AHA Guidelines for the Diagnosis and Management of Heart Failure in Adults: A Report of the American College of Cardiology Foundation/American Heart Association Task Force on Practice Guidelines Developed in Collaboration with the International Society for Heart and Lung Transplantation. Journal of the American College of Cardiology, 53, 1343-1382. http://dx.doi.org/10.1016/j.jacc.2008.11.009

[15] Makkar, R.R., Fromm, B.S., Steinman, R.T., et al. (1993) Female Gender as a Risk Factor for Torsades de Pointes Associated with Cardiovascular Drugs. JAMA, 270, 2590-2597. http://dx.doi.org/10.1001/jama.1993.03510210076031

[16] Li, L., Liu, H.R., Shu, J.L., et al. (2007) Clinical Investigation of Q-T Prolongation in Hepatic Cirrhosis. Zhonghua Yi Xиe Za Zhi, 87, 2717-2718.

[17] Bal, J.S. and Thuluvath, P.J. (2003) Prolongation of QTc Interval: Relationship with Etiology and Severity of Liver Disease, Mortality and Liver Transplantation. Liver International, 23, 243-248. http://dx.doi.org/10.1034/j.1600-0676.2003.00833.x

[18] Kosar, F., Ates, F., Sahin, I., et al. (2007) QT Interval Analysis in Patients with Chronic Liver Disease: A Prospective Study. Angiology, 58, 218-24. http://dx.doi.org/10.1177/0003319707300368 